ARTICLE

DOI: $10.1038 / \mathrm{s} 41467-018-04416-0$

\title{
CRISPR/Cas9-mediated gene targeting in Arabidopsis using sequential transformation
}

\author{
Daisuke Miki ${ }^{1}$, Wenxin Zhang ${ }^{1,2}$, Wenjie Zeng ${ }^{1,2}$, Zhengyan Feng ${ }^{1}$ \& Jian-Kang Zhu ${ }^{1,3}$
}

Homologous recombination-based gene targeting is a powerful tool for precise genome modification and has been widely used in organisms ranging from yeast to higher organisms such as Drosophila and mouse. However, gene targeting in higher plants, including the most widely used model plant Arabidopsis thaliana, remains challenging. Here we report a sequential transformation method for gene targeting in Arabidopsis. We find that parental lines expressing the bacterial endonuclease Cas9 from the egg cell- and early embryospecific DD45 gene promoter can improve the frequency of single-guide RNA-targeted gene knock-ins and sequence replacements via homologous recombination at several endogenous sites in the Arabidopsis genome. These heritable gene targeting can be identified by regular PCR. Our approach enables routine and fine manipulation of the Arabidopsis genome.

\footnotetext{
${ }^{1}$ Shanghai Center for Plant Stress Biology and Center for Excellence in Molecular Plant Sciences, Chinese Academy of Sciences, 200032 Shanghai, China. ${ }^{2}$ University of Chinese Academy of Sciences, 100049 Beijing, China. ${ }^{3}$ Department of Horticulture and Landscape Architecture, Purdue University, West Lafayette, IN 47907, USA. These authors contributed equally: Daisuke Miki, Wenxin Zhang. Correspondence and requests for materials should be addressed to D.M. (email: miki@sibs.ac.cn) or to J.-K.Z. (email: jkzhu@sibs.ac.cn)
} 
recise genome modification such as DNA knock-in and gene replacement (i.e., gene targeting) via homologous recombination is a powerful tool that is widely applied for research in many organisms, including Drosophila and animals ${ }^{1-3}$. However, gene targeting (GT) is still very challenging in higher plant species, because of low efficiency of homologous recombination ${ }^{4}$.

Engineered sequence-specific nucleases such as zinc finger nucleases (ZFNs), transcription activator-like effector nucleases (TALENs) and clustered regularly interspaced short palindromic repeats (CRISPR)/CRISPR-associated protein 9 (Cas9) have been used to generate site-specific double stranded breaks (DSBs) for genome editing in numerous organisms ${ }^{1,5-7}$. Repair of these DSBs via error-prone non-homologous end-joining (NHEJ) leads to random mutations, whereas error-free homology-directed repair (HDR) creates precise sequence changes when a homologous DNA substrate is provided. A goal of genome editing is to achieve heritable GT, defined as the precise insertion or replacement of sequence at any genomic locus of interest in germline cells.

However, HDR-mediated GT at endogenous genes is extremely inefficient in higher plants, preventing its widespread application $^{4}$. The first GT in plants was demonstrated at a kanamycin resistance gene in tobacco, with a frequency ranging from $10^{-3}$ to $10^{-6}$ (refs. ${ }^{8,9}$ ). A higher efficiency method using positive - negative selection was later developed in rice ${ }^{10}$; however, this complicated strategy has been used to modify only several genes in rice ${ }^{11}$ and has not been successfully applied to other plants, including Arabidopsis ${ }^{12,13}$ and tobacco ${ }^{14}$. Sequence-specific nucleases can increase the efficiency of $\mathrm{GT}^{1,15,16}$, and CRISPR/ Cas9-assisted HDR has been used for GT in various model systems, including human stem cells ${ }^{15}$. The introduction of DSBs also increased the frequency of HDR in plants ${ }^{17,18}$, and recent publications report using sequence-specific nucleases for HDRmediated GT in Arabidopsis ${ }^{19-24}$, tobacco ${ }^{25-30}$, soybean ${ }^{31}$, tomato $^{32,33}$, rice $^{34-41}$, maize ${ }^{42-46}$, wheat ${ }^{47,48}$, potato ${ }^{49}$, barley ${ }^{50}$, flax $^{51}$, and cotton ${ }^{52}$. Nevertheless, these GT events mostly relied on selection for antibiotic or herbicide resistance genes at the targeted loci to improve efficiency. The few GT events that did not rely on selection markers displayed extremely low frequencies $^{24,31,43}$, thus limiting the usefulness of these methods.

Here, we describe a simple method for seamless GT in Arabidopsis, including in-frame gene knock-ins and amino acid substitutions. We demonstrate the utility of our method by targeting the endogenous DNA glycosylase genes ROS1 and DME in Arabidopsis.

\section{Results}

Inefficient GT by an all-in-one strategy. To achieve efficient GT in Arabidopsis, we first designed an "all-in-one" T-DNA construct that contains: (i) Cas9 driven by the CaMV $35 \mathrm{~S}$ promoter (35Spro::Cas9), (ii) an sgRNA driven by the AtU6 promoter, that targets a site near the stop codon of ROS1, and (iii) a donor DNA fragment for in-frame GFP knock-in (Supplementary Fig. 1a). We screened T1 plants by PCR (e.g. Supplementary Fig. 1b), and identified 2/30 with a positive GT signal (Supplementary Table 1). In contrast, a control construct without an sgRNA did not yield any T1 plants with a positive GT signal (Supplementary Table 1). Neither of the T1-positive plants gave rise to T2 progenies with a positive GT signal, although bulk screening of 18 remaining T2 lines identified a positive GT signal (Supplementary Table 1). Southern blot analysis of individual plants from this PCR-positive T2 population failed to detect any GT-positive plants (Supplementary Fig. 1b), suggesting that the GT-positive PCR signal may have come from a small number of somatic cells. Thus, this method did not generate heritable GT. A similar all-in-one construct also failed to generate heritable in-frame ROS1-Luc knock-ins (Supplementary Table 1).

The expression of Cas9 under germline-specific promoters was recently shown to increase the efficiency of CRISPR/Cas9mediated gene editing in Arabidopsis ${ }^{53-55}$. We hypothesized that driving Cas9 expression from a germline-specific promoter instead of the CaMV 35S promoter might increase the frequency of heritable GT. We tested the following promoters: the egg celland early embryo-specific promoter DD45 $53,54,56$, the pollenspecific promoter Lat $52^{53}$, and the shoot apical meristem-active promoters $\mathrm{YAO}^{55}$ and $\mathrm{CDC} 45^{57}$. We generated all-in-one constructs for GFP knock-in into the GLABRA2 (GL2) locus, utilizing these promoters to drive Cas9 expression and an sgRNA known to efficiently generate site-specific DSB in $G L 2^{53}$ (Supplementary Fig. 1c). Although we observed high frequencies of GT-positive PCR signals with some of these all-in-one constructs, we did not identify any heritable GT lines (Supplementary Fig. 1d-i, Supplementary Table 2). Sequencing of the PCR products indicated that precise GT events occurred, but they likely represent minor events in some somatic cells. Thus, although expression of Cas 9 under these specific promoters might improve GT efficiency in some somatic tissues, it did not lead to heritable GT.

Knock-in into the ROS1 locus by sequential transformation. Next, we used a "sequential transformation method" to evaluate GT efficiency ${ }^{35,41}$ in parental Arabidopsis plants that already express Cas9 from a germline-specific (DD45, Lat52, YAO or CDC45) promoter (Fig. 1). These parental Cas9 lines also express a GL2-targeting sgRNA from the AtU6 promoter. We used the two highest efficiency CRISPR/Cas9 lines, which were screened from 32 to 36 independent T1 lines based on the mutation rates at the GL2 locus, for each specific promoter ${ }^{53}$. We used these Cas9-expressing plants as parental lines for new transformations with a construct containing: (i) HDR donor sequence, (ii) sgRNA targeting a genomic locus of interest, (iii) a selectable marker for plants that are positive for the donor construct (Figs. 1, 2a). The new transformation T1 transgenic plants were selected using the Basta resistance gene. These T1 plants express Cas9 and a specific sgRNA, and contain a specific HDR donor sequence. T1 seeds were harvested and germinated without selection on MS plates; 20-30 of the resulting T2 seedlings were subsequently pooled together, and GT events were analyzed by PCR in bulk. Further, another batch of T2 plants from the bulk positive lines were investigated as individual plants (Fig. 1).

Transformation of a construct containing ROS1-targeting sgRNA and ROS1-GFP donor sequence into DD45pro::Cas9 lines \#58 and \#70, but not other promoter::Cas9 lines, gave rise to Southern blot- and PCR-positive GT signals (Fig. 2a-c, Table 1, Supplementary Fig. 2, Supplementary Table 3). Six out of 11 tested plants from two T2 populations in the DD45-\#58 background were homozygous ROS1-GFP GT lines based on Southern blot analysis, and 2 of 12 tested plants from another two T2 populations in the DD45-\#70 background were homozygous (Table 1; e.g. Fig. 2c). Sanger sequencing confirmed that there were no mutations in the $5^{\prime}$ and $3^{\prime}$ homology arms and their border regions, and that GFP integration downstream of the ROS1 gene was in-frame (Supplementary Figs. 4a and 5a). We examined the progenies of a heterozygous T2 GT plant and found that the integrated ROS1-GFP segregated in T3 (Fig. 2d). We analyzed mRNA expression in these T3 plant samples by RT-PCR and qRT-PCR, and observed comparable expression of the ROS1GFP knock-in with endogenous ROS1 (Fig. 2e, f). Further, the root tissues of homozygous T3 ROS1-GFP plants displayed GFP 
Parental line (ref. 53) (Hygromycin selection)
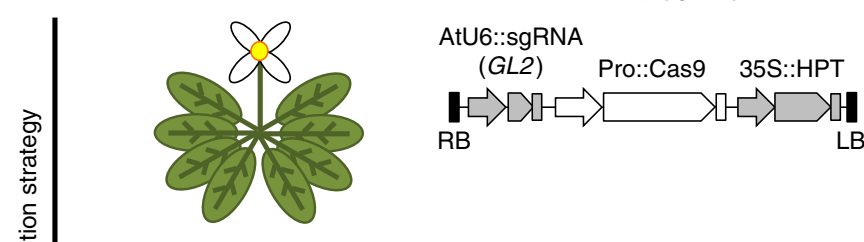

Agrobacterium transformation with construct containing gRNA and donor sequence
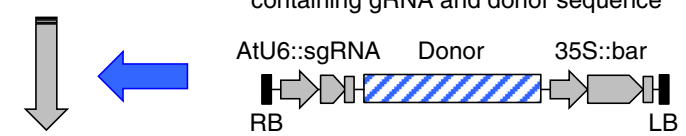

$\mathrm{RB}$
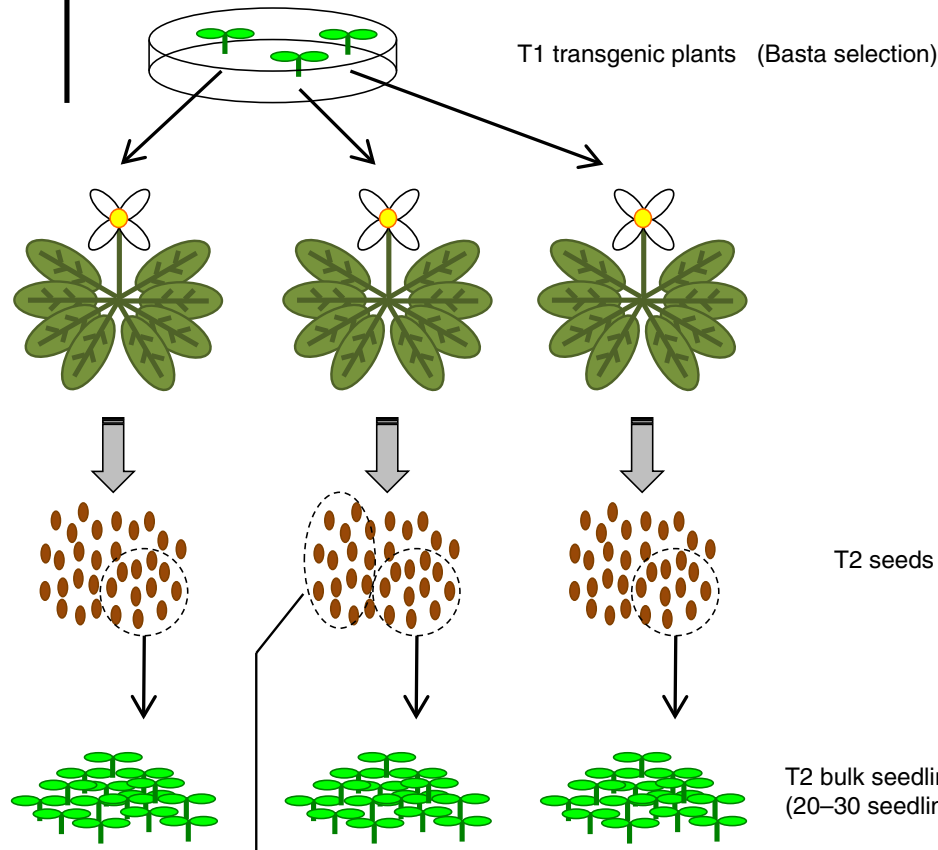

T2 seeds
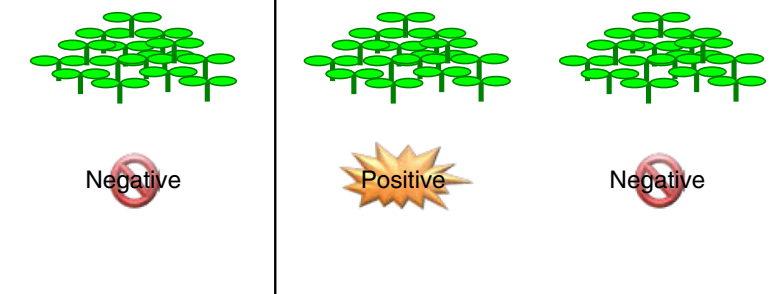

T2 bulk seedlings

(Without

(20-30 seedlings) antibiotic selection)
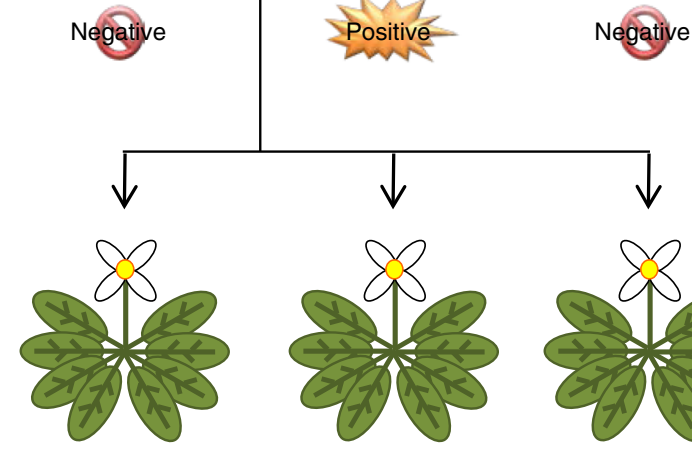

PCR screening antibiotic selection)
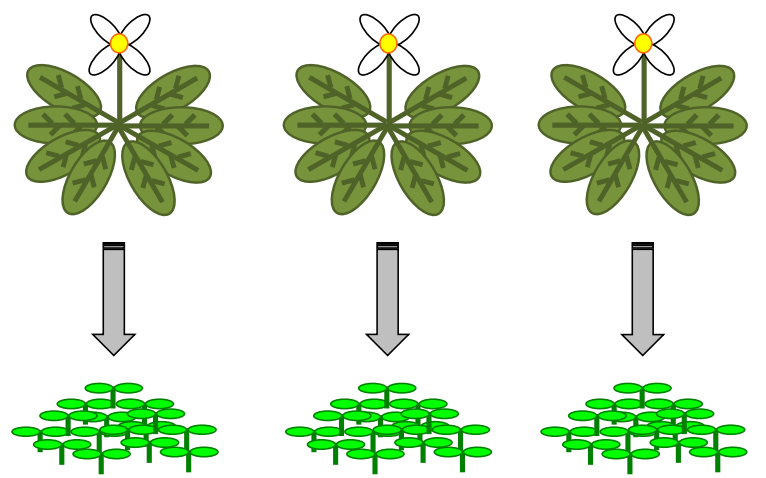

T2 individual plants

(Without antibiotic selection)

Fig. 1 Outline of the sequential transformation strategy for gene targeting and screening procedure. HDR donor constructs containing a selection marker and sgRNAs targeting genes of interest were transformed into parental lines, which were already transgenic for an incidental GL2-targeting sgRNA and, importantly, for Cas9 driven by a specific promoter. T1 transgenic lines were selected with Basta, and T2 seedlings were obtained and analyzed in bulk. The positive lines were used for further experiments with individual T2 plants. Portions of the images were obtained from the Microsoft PowerPoint clip art data base 
a

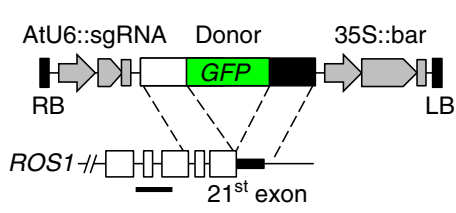

b

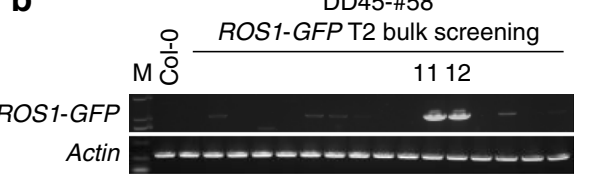

C
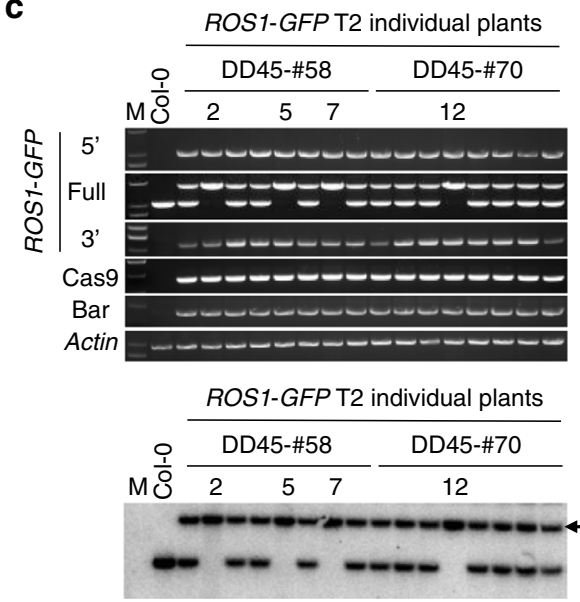

d

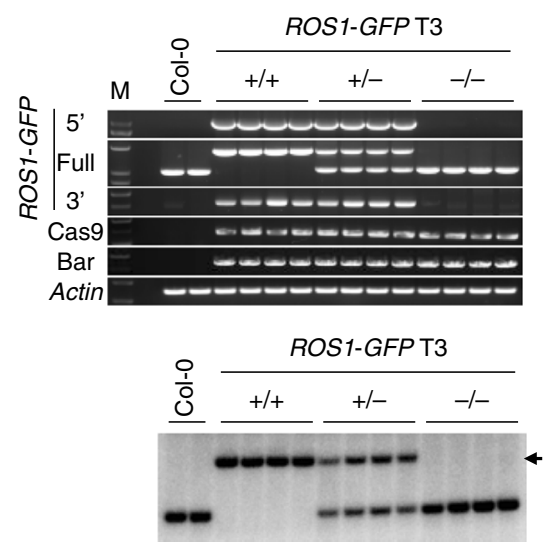

e
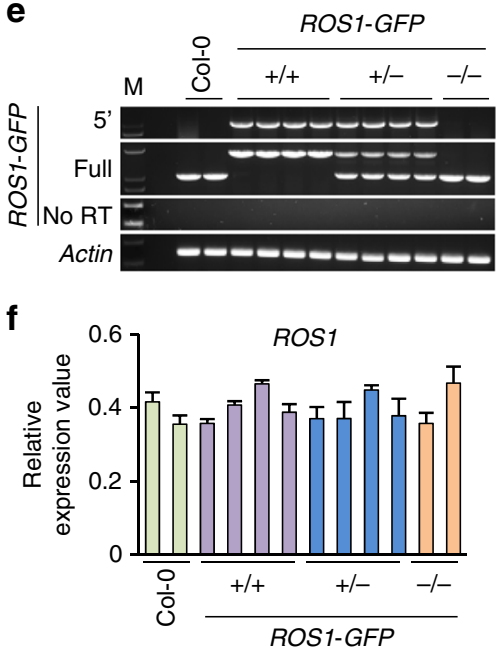

g

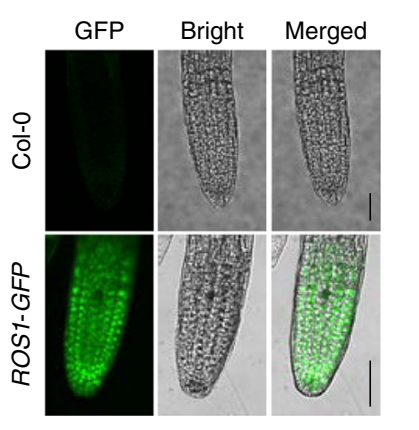

h

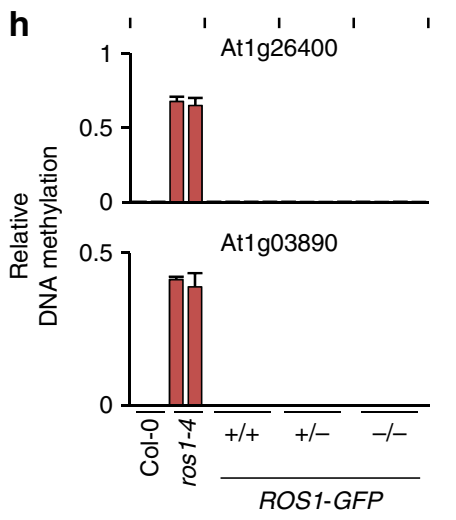

i
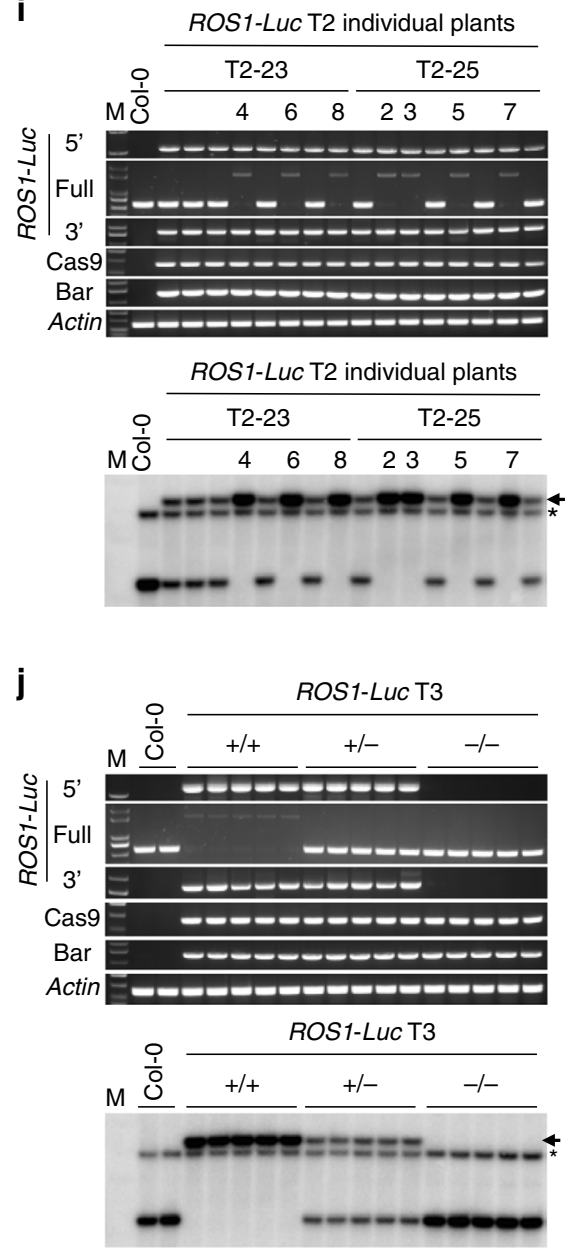

$\mathbf{k}$

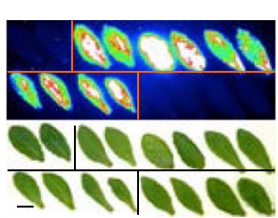

Col-0 $\mid$ ROS1-LuC (+/+)

ROS1-LuC (+/-) $\mid$ ROS1-LuC (-/-)

Fig. 2 GFP integration into the endogenous ROS1 locus by gene targeting. a Schematics showing HDR donor transgene construct (top) and part of the targeted ROS1 locus (bottom). The horizontal line indicates the position of a probe for Southern blot hybridization. b PCR screening of T2 bulk samples (see Supplementary Figure 2). Primers for the $5^{\prime}$ part of ROS1-GFP were used (see Supplementary Figure 2). c, d Genotyping PCR and Southern blotting of a subset of individual T2 (c) and T3 plants (d) of ROS1-GFP, respectively. The eight plants in the DD45-\#58 background were selected from populations 11 and 12 in (b). Arrow indicates the band of ROS1-GFP from gene targeting. e RT-PCR and $\mathbf{f}$ qRT-PCR on the same T3 plants as in (d). The error bars indicate $P$ values of Student's $t$ test $(n=4)$. See Supplementary Figure 2. $\mathbf{g}$ Detection of GFP fluorescence. Scale bar, $50 \mu \mathrm{m}$. $\mathbf{h}$ qChop-PCR at the At1g26400 and At1g03890 loci. The ros1-4 mutant was used as a positive control. The error bars indicate $P$ values of Student's $t$ test $(n=4)$. i, j Genotyping PCR and Southern blotting for individual T2 lines (i) and T3 plants (j) of ROS1-Luc, respectively. Arrow indicates the band of ROS1-Luc from gene targeting, and the asterisk denotes non-specific cross-hybridization band. $\mathbf{k}$ Luminescence in T3 ROS1-Luc leaves. Scale bar, $1 \mathrm{~cm}$. All PCR primers are as depicted in Supplementary Figure 2b, c and Supplementary Table 4

fluorescence (Fig. 2g). To determine whether the ROS1-GFP knock-in retained ROS1 function, we assessed the DNA methylation level of two genomic loci known to become hypermethylated in loss-of-function ros 1 mutant plants by quantitative Chop-PCR (Fig. 2h) ${ }^{58}$. Homozygous T3 ROS1-GFP knock-in plants did not display hypermethylation at these loci, suggesting that the in-frame integration of GFP did not interfere with ROS1 function, and that the ROS1-GFP was functional.
Thus, our sequential transformation method efficiently generates precise and heritable GT.

Next we tested whether a fragment longer than GFP could be integrated at the ROS1 locus. We used the same sgRNA and homology arms to make a donor construct that contained firefly luciferase (Luc: $1653 \mathrm{bp}$ ) instead of GFP (720 bp), and transformed the construct into parental CRISPR/Cas9 lines. Two positive GT lines were identified in T2 bulk screening by 


\begin{tabular}{|c|c|c|c|c|c|c|c|}
\hline \multirow[t]{2}{*}{ Construct } & \multirow[t]{2}{*}{ Parental line } & \multicolumn{6}{|l|}{ T2 } \\
\hline & & PCR & Line name & PCR & Southern & Homo & GT efficiency \\
\hline \multirow[t]{2}{*}{ ROS1-GFP } & DD45-\#58 & $2 / 26$ & T2-11 & $7 / 56$ & $7 / 7$ & 4 & $7.7 \%(2 / 26)$ \\
\hline & & & $\mathrm{T} 2-12$ & $4 / 59$ & $4 / 4$ & 2 & \\
\hline \multirow[t]{2}{*}{ ROST-Luc } & DD45-\#58 & $2 / 32$ & $\mathrm{~T} 2-23$ & $20 / 65$ & $20 / 20$ & 3 & $6.3 \%(2 / 32)$ \\
\hline & & & $\mathrm{T} 2-25$ & $10 / 72$ & $10 / 10$ & 4 & \\
\hline \multirow[t]{2}{*}{ DME-GFP } & DD45-\#58 & $2 / 22$ & $\mathrm{~T} 2-9$ & $20 / 60$ & $20 / 20$ & 16 & $9.1 \%(2 / 22)$ \\
\hline & & & $\mathrm{T} 2-14$ & $42 / 60$ & $42 / 42$ & 15 & \\
\hline \multirow[t]{2}{*}{ GFP-DME } & DD45-\#58 & $2 / 24$ & $\mathrm{~T} 2-11$ & $4 / 57$ & $4 / 4$ & 1 & $8.3 \%(2 / 24)$ \\
\hline & & & $\mathrm{T} 2-24$ & $11 / 60$ & $6 / 6$ & 3 & \\
\hline
\end{tabular}

PCR, and precise knock-in was confirmed in individual T2 and T3 plants (Table 1, Figs. 1, 2i, j). These true GT-positive (PCR and Southern blotting positive in individual T2 plants) ROS1-Luc lines were all from the DD45pro::Cas9 background (Table 1, Supplementary Table 3). The leaves of homozygous and heterozygous ROS1-Luc T3 plants displayed luminescence signals, unlike those from control plants without GT (Fig. 2k). Thus, a fragment as large as $1.6 \mathrm{~kb}$ can be stably integrated into a genomic locus using our sequential transformation GT strategy.

Knock-in into the $D M E$ locus. Next, to investigate the broad utility of our GT method, we attempted to generate in-frame GFP knock-ins at the $5^{\prime}$ end and the $3^{\prime}$ end of DME (At5g04560), a DNA glycosylase gene on a different chromosome than ROS1 in Arabidopsis. We designed specific sgRNAs and donor constructs for a $3^{\prime}$ in-frame fusion (DME-GFP) and $5^{\prime}$ in-frame fusion (GFP-DME) (Fig. 3a, b, Supplementary Fig. 3). The sgRNA used to generate GFP-DME also targets the $3^{\prime}$ homology region of the donor construct, so we introduced silent mutations within the $3^{\prime}$ donor sequence of GFP-DME to prevent sgRNA binding, DSB and mutations following precise knock-in (Supplementary Fig. 3b).

These T-DNA constructs were transformed into the parental Lat52, YAO, CDC45, and DD45 promoter-driven CRISPR/Cas9 lines. Although some GT signals were detected by PCR in the T1 and T2 plants from the Lat52, YAO and CDC45 parental lines, they were not heritable GT events, given that positive signals were not detected by Southern blotting or in some cases even by PCR in individual T2 plants (Supplementary Table 3). In contrast, true GT-positive (PCR and Southern blotting positive in individual T2 plants) signals were detected for DME-GFP from 2 out of $22 \mathrm{~T} 2$ populations $(9.1 \%)$ in the DD45-\#58 parental line (Table 1). Further, two positive GT signals were detected for GFP-DME from 24 T2 populations (8.3\%), from each of the DD45-\#58 and DD45-\#70 parental lines (Table 1). Analysis of individual T2 plants revealed homozygous and heterozygous plants for both $D M E-G F P$ and GFP-DME fusions (Fig. 3c, d, Supplementary Fig. 3). The heterozygous T2 plants segregated in T3 (Fig. 3e, f, Supplementary Fig. 3). These in-frame GFP knock-ins at the $5^{\prime}$ and $3^{\prime}$ ends of DME were confirmed by sequencing the PCR products (Supplementary Figs. $4 \mathrm{~b}, \mathrm{c}, 5 \mathrm{~b}, \mathrm{c}$ ).

Homozygous and heterozygous DME-GFP and GFP-DME plants did not show any developmental or growth defects, suggesting that the gene-targeted $D M E$ is functional, since dme loss-of-function mutants show maternal lethality ${ }^{59}$. To further confirm that the DME-GFP and GFP-DME in-frame fusion proteins are functional, we examined the seed abortion ratios of homozygous DME-GFP and GFP-DME T3 plants, and found that they were comparable with that of wild-type Col-0 plants (Fig. 3g). Thus, the DME-GFP and GFP-DME in-frame fusions are functional.

Sequence replacement at the $D M E$ locus. An important goal of GT is the fine manipulation of endogenous genes by gene replacement. To test the feasibility of gene replacement, we attempted to substitute an amino acid within a conserved motif of $D M E$ (Supplementary Fig. 6). The Fe-S motif is highly conserved in the family of 5-methylcytosine DNA glycosylases, and is required for 5-methylcytosine DNA glycosylase activity of DME and ROS1 in vitro ${ }^{60,61}$. We generated mutated forms of a DME donor by changing a conserved proline to alanine (P1633A) and phenylalanine to alanine (F1648A). Silent mutations were also integrated at the PAM sequence to block additional DSBs, following the CORRECT method ${ }^{15}$ (Supplementary Fig. 6). The two constructs containing the mutated DME donors and corresponding sgRNAs were transformed into YAO, CDC45, and DD45 promoter-driven CRISPR/Cas9 parental lines. We used a PCR-restriction enzyme assay to uncover amino acid substitution GT events. Heritable GT lines were obtained only in the DD45pro::Cas9 parental background (Fig. 4a, b, Table 2, Supplementary Table 3). We sequenced the PCR amplicons from GT-positive T2 plants and found accurate amino acid substitutions, with no other mutations (Fig. 4c, d). Southern blot analysis of several T3 plants revealed that they were all heterozygous for the amino acid substitution GT (Fig. 4e). Thus, the amino acid substitution GT was stable and heritable.

We did not obtain any homozygous P1633A and F1648A GT plants in T2 or T3 generations, likely due to the lethality of lossof-function dme mutations ${ }^{59}$. Indeed, approximately $50 \%$ of the seeds of the P1633A and F1648A heterozygous T3 plants aborted, whereas no seed abortion was found in T3 plants without the amino acid substitution GT (Fig. 4f). Thus, these two highly conserved amino acids within the Fe-S motif, P1633 and F1648, are essential for $D M E$ function in vivo.

GT effect on DNA methylation. ZFN-mediated GT of the endogenous locus $P P O X$ in plants reportedly alters its epigenetic status ${ }^{62}$. We performed individual locus bisulfite 
a

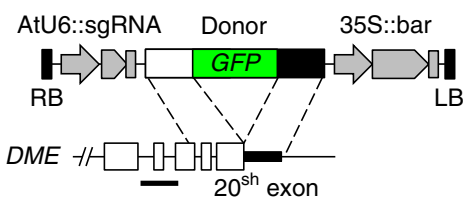

C

DD45-\#58

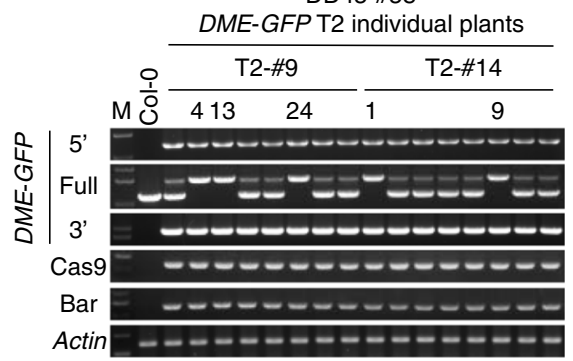

DD45-\#58

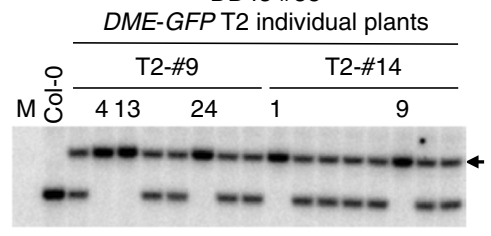

e
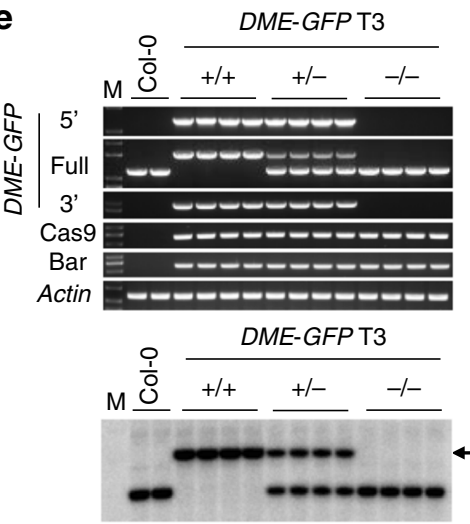

b

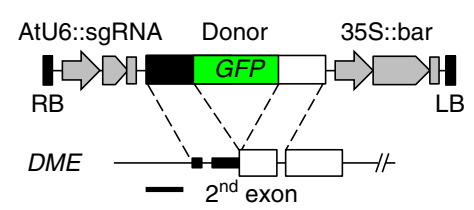

d

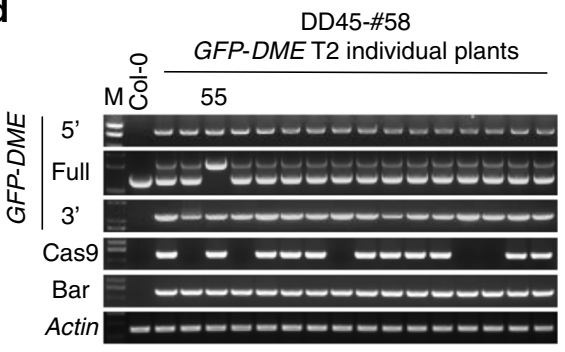

DD45-\#58

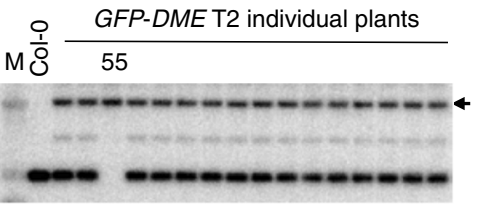

$\mathbf{f}$

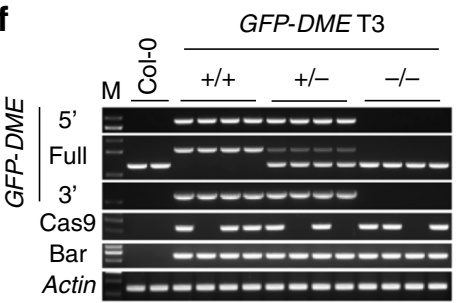

GFP-DMET3

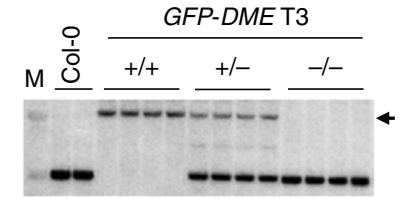

g

Col-0 $98.76 \pm 0.87 \%(n=3)$
DME-GFP $99.09 \pm 1.08 \%(n=9)$
$98.94 \pm 1.06 \%(n=6)$

Fig. 3 GFP knock-in into the endogenous DME locus by gene targeting. $\mathbf{a}$, b Schematics showing HDR donor transgene constructs and part of the targeted DME locus for DME-GFP and GFP-DME knock-in, respectively. The horizontal lines indicate the positions of probes for Southern blotting. c-f Genotyping PCR and Southern blotting for individual T2 lines (b) and T3 plants (e) of DME-GFP, respectively. Arrow indicates the band of DME-GFP from gene targeting (see Supplementary Figure 3). Genotyping PCR and Southern blotting for individual T2 lines (d) and T3 plants (f) of GFP-DME, respectively. Arrow indicates the band of GFP-DME from gene targeting (see Supplementary Figure 3). $\mathbf{g}$ Analysis of seed abortion. Seeds from Col-0, homozygous DME-GFP, and GFP-DME knock-in T3 plants were analyzed. Scale bar, $1 \mathrm{~mm}$. All PCR primers are as depicted in Supplementary Figure 3 and Supplementary Table 4

sequencing to analyze whether DNA methylation is affected in two independent homozygous T4 ROS1-GFP GT plants generated by our sequential GT strategy. We did not observe substantial changes in cytosine methylation in either the $5^{\prime}$ or $3^{\prime}$ homology arm regions (Supplementary Fig. 7), suggesting that our GT method did not affect the DNA methylation status of the targeted genomic locus.

\section{Discussion}

Using our new approach for efficient and heritable GT in Arabidopsis, we achieved precise knock-ins, generating ROS1-GFP, ROS1-Luc, DME-GFP, and GFP-DME fusions, as well as gene replacements, generating $P 1633 A$ and $F 1648 A$ amino acid substitutions in DME. Only parental plant lines expressing Cas9 under the egg cell- and early embryo-specific promoter DD45 gave rise to efficient and heritable GT, without any need for a selection marker at the targeted locus. The fact that only DD45 promoter-driven Cas9 lines yielded heritable GT suggests that HDR may be more efficient in egg cells and/or early embryos than in other germline tissues (e.g., pollen and shoot apical meristem). We propose that germline GT occurs immediately after transformation, when Agrobacteria enter the Cas9expressing ovule ${ }^{63}$ to deliver the T-DNA containing sgRNA and donor DNA. Efficient HDR may occur in the egg cell and/or very early embryo, perhaps before T-DNA integration. 
a

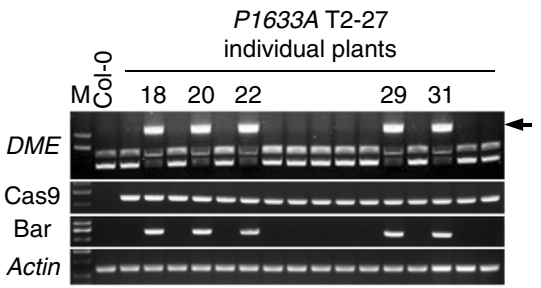

c

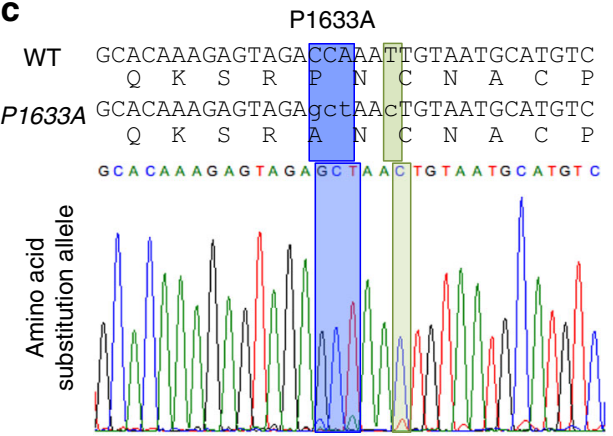

WT

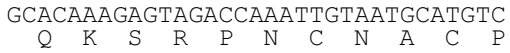

GCACAAAGAGTAGACCAAATT GTAAT GCAT GTC

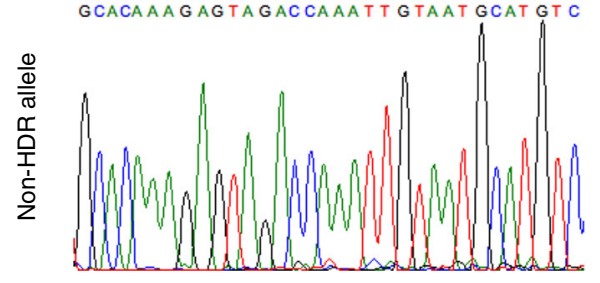

b

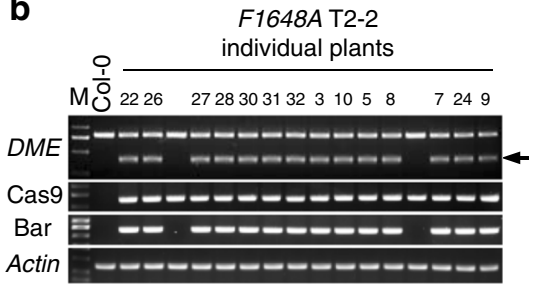

d

F1648A

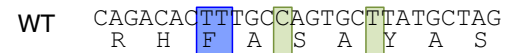

F1648A CAGACAdgctgCtAgtgCatatgCTAG

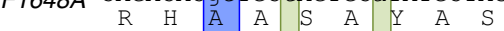

CAGACACGGTGCTAG TG CAATATGCTAG

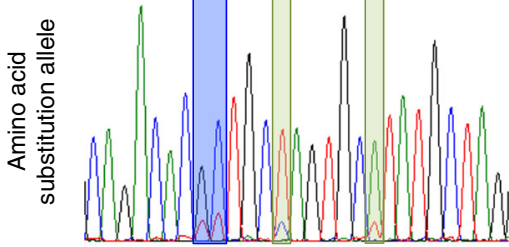

WT CAGACACTTTGCCAGTGCTTATGCTAG

CAGACACTTTGCCAGTGCTTATGCTAG

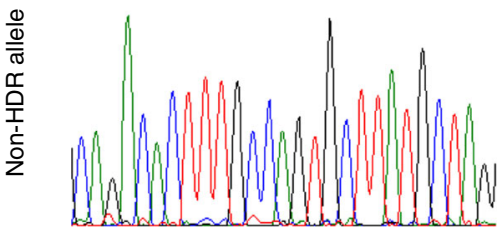

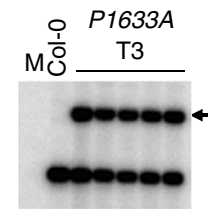

f

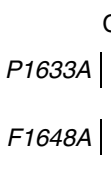

Col-0
$\mid \begin{aligned} & +/- \\ & -/- \\ & \mid+- \\ & -/-\end{aligned}$

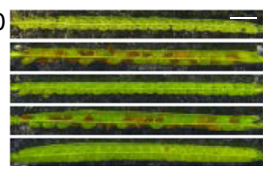

$98.21 \pm 1.93 \%(n=6)$

$50.70 \pm 7.09 \%(n=14)$

$98.71 \pm 1.47 \%(n=6)$

$48.50 \pm 6.27 \%(n=10)$

$98.79 \pm 1.21 \%(n=4)$

Fig. 4 Single amino acid substitution at the endogenous DME locus by gene targeting. a, b Genotyping of T2 individual plants for P1633A and F1648A, respectively. Arrows indicate the specific sequence substitution as detected by PCR and restriction enzyme digestion. PCR primers are as depicted in Supplementary Figure 6a and Supplementary Table 4. Detailed information is described in Supplementary Figure 6. c, d Sequence confirmation of the P1633A and F1648A substitutions, respectively, in individual T2 plants. Blue highlights indicate amino acid substitution, green highlights indicate silent mutations. The sequence chromatograms are taken from FinchTV. e Southern blotting. The P1633A substitutions were confirmed by Southern blotting in heterozygous T3 plants. The arrow indicates the band caused by specific base substitution. $\mathbf{f}$ Analysis of seed abortion. Seeds from Col-0, heterozygous GT $(+/-)$, and non-GT (-/-) P1633A and F1648A T3 plants were analyzed. Scale bar, $1 \mathrm{~mm}$

\section{Table 2 Gene replacement efficiencies for the DME locus}

\begin{tabular}{|c|c|c|c|c|c|c|c|}
\hline \multirow[t]{3}{*}{ Construct } & \multirow[t]{3}{*}{ Parental line } & \multicolumn{6}{|l|}{ T2 } \\
\hline & & \multicolumn{2}{|l|}{ Bulk } & \multicolumn{3}{|c|}{ Individual plants } & \multirow[t]{2}{*}{ GT efficiency } \\
\hline & & PCR & Sequencing & Line name & PCR & Sequencing & \\
\hline DME-P1633A & DD45-\#45 & $19 / 35$ & $1 / 19$ & $\mathrm{~T} 2-27$ & $6 / 54$ & $6 / 6$ & $5.3 \%(1 / 19)$ \\
\hline DME-F1648A & DD45-\#45 & $1 / 1$ & $1 / 1$ & T2-2 & $53 / 60$ & $40 / 40$ & $100 \%(1 / 1)$ \\
\hline
\end{tabular}

T2 bulk seedlings were genotyped by restriction digestion and PCR, and the positive samples were sequenced. For DME-P1633A, one of the 19 T2 bulk PCR positives had the P1633A amino acid substitution, and the rest had errors/mutations due to NHEJ. Fifty-four individual T2 plants from the positive line in T2 bulk screening were analyzed by restriction digestion and PCR, and six were found positive, and sequencing confirmed that all six had the P1633A substitution. For DME-F1648A, only one T2 population was obtained, which was found positive in the T2 bulk restriction digestion-PCR assay and sequencing showed that it was caused by F1648A substitution. Sixty T2 individual plants from this population were analyzed by restriction digestion-PCR and 53 were found positive. Forty of the 53 were tested by sequencing and all 40 were confirmed to have the F1648A substitution

Alternatively, HDR and the resulting GT may occur during the reproductive stage of $\mathrm{T} 1$ plants, when the T-DNA is already stably integrated. Five GFP-DME heterozygous T2 plants showed segregation from the Cas 9 transgene (Fig. 3e, f), indicating that heritable knock-in occurred in T1 plants. The frequency of GT- positive plants in T2 populations ranged from 4/59 to 53/60 (Tables 1 and 2). The data are consistent with heritable GT events occurring in early embryos following the new transformation, in agreement with the strong activity of DD45 promoter in egg cells and early embryos ${ }^{56}$. 
All of the heritable GT events we observed were precise, without unexpected mutations or rearrangements at the target sites. The GT efficiency by our method was $5.3 \%$ for $D M E$ $P 1633 A$ and was higher for other knock-ins or gene replacement (Tables 1 and 2). We analyzed T2 bulk DNA to determine whether the T-DNA copy numbers may contribute to efficient GT. Our results show that GT events were not related to T-DNA copy numbers of Cas9 or of the HDR donor transgene (Supplementary Fig. 8), suggesting that other unknown factors might be important. Additional research is required to understand and improve GT efficiency, and to apply this GT method to other plants including crops.

Here we revealed heritable GT and simple PCR-based identification, without the need of any selection marker at the target locus. This approach enables routine GT in Arabidopsis. Using egg cell- and early embryo-specific promoters to drive the expression of Cas 9 or other site-specific nucleases, in combination with strategies for the effective delivery of donor DNA (such as described in ref. ${ }^{4}$ ), might lead to efficient GT technologies in other plants, including crop plants.

\section{Methods}

Gene accession numbers. ROS1, At2g36490; DME, At5g04560; GL2, At1g79840.

Plant materials and growth condition. The Arabidopsis thaliana accession Col-0 was used for all experiments. All plants were grown at $22^{\circ} \mathrm{C}$ on half Murashige and Skoog (MS) medium with $1 \%$ sucrose or in soil with a $16 \mathrm{~h} \mathrm{light} / 8 \mathrm{~h}$ dark photoperiod. Parental T2 plants ${ }^{53}$ were selected on the hygromycin $(25 \mathrm{mg} / \mathrm{L})$ containing MS plates for 10 days, then transplant in soil. The new transformation T1 lines were directly sowed in soil, and selected by three times Basta spray.

Plasmid construction. The optimized coding sequence of hSpCas9 (CRISPR/Cas9) plasmids for GL2 GT, which were already reported ${ }^{53}$, were constructed in pCambia1300. For all-in-one GT constructs, donor sequence was added to the published CRISPR/Cas9 constructs. For GT constructs for the sequential transformation strategy, AtU6 promoter-driven sgRNA and donor sequence were constructed in pCambia3301. All transformants were generated by the flower dipping method.

DNA analysis. Total DNA was extracted by the cethyltrimethyl ammonium bromide (CTAB) method from 10-day-old seedling for bulk analysis or 4- to 6week-old for individual plant analysis. Extracted DNA was used for analysis of GT events by PCR and Southern blotting. Southern blotting was performed according to published protocols. Briefly, extracted DNA was digested overnight with chosen restriction enzymes, then separated on a $1.5 \%$ agarose gel, visualized by Image Lab Software and Gel Doc XR (BIO-RAD), and then transferred to nylon membrane (GE Healthcare). The probes were labeled with 32P- $\alpha$-dCTP by using the Random primer DNA labeling kit (Takara). The hybridization signals were detected with a phosphor imager (Fuji). Un-cropped images of the most important Southern blots were supplied as Supplementary Fig. 10.

RNA analysis. For RT- and qRT-PCR, total RNA was extracted form 10-day-old or 4-week-old plants by using RNeasy Plant mini kit (Qiagen), treated with Turbo DNA-free (Ambion), and reverse transcribed by TransScript II (TransGen Biotech) with oligo (dT) primer. Then $1 \mu \mathrm{L}$ of RT product was used as template for expression analysis. The raw data of some of the $\mathrm{qPCR}$ analysis are shown in Supplemental Fig. 9

Detection of GFP fluorescence and Luc luminescence. GFP signal was observed in the roots of 3-day-old seedlings by confocal microscopy (Leica TCS SP8). Bright field and GFP fluorescence images were merged using ImageJ.

To determine firefly luciferase (Luc) reporter activity, $0.5 \mu \mathrm{M}$ luciferin (Promega) in $0.01 \%$ Triton X-100 was sprayed onto 4 -week-old mature leaves, followed by luminescence imaging using a high-performance CCD camera.

DNA methylation analysis. DNA methylation was analyzed by bisulfite sequencing. Total DNA was extracted using the CTAB method, and un-methylated cytosines were converted into uracil by using EZ DNA Methylation-Gold Kit (ZYMO RESEARCH). Genomic regions of interest were amplified by specific primers (Supplementary Table 4), then the amplicons were cloned into pMD-18 (Takara), and at least 27 independent colonies were sequenced. The sequence results were analyzed by Kismeth.
Data availability. The authors declare that all the data supporting the findings of this study are available within the paper and its supplementary information files. The data sets generated or analyzed during the current study are available from the corresponding author on reasonable request. We deposited our DD45::CRSIPR/ Cas9 parental lines, DD45-\#58 and DD45-\#70, to the Arabidopsis Biological Resource Center (ABRC). The seeds were assigned the stock numbers CS69955 and CS69956, respectively. The two homozygous DD45::CRISPR/Cas9 parental lines could retain a high rate of GT when they are propagated to future generations with hygromycin selection.

Received: 6 December 2017 Accepted: 26 April 2018

Published online: 17 May 2018

\section{References}

1. Chandrasegaran, S. \& Carroll, D. Origins of programmable nucleases for genome engineering. J. Mol. Biol. 428, 963-989 (2016).

2. Hinnen, A., Hicks, J. B. \& Fink, G. R. Transformation of Yeast. Proc. Natl. Acad. Sci. USA 75, 1929-1933 (1978).

3. Thomas, K. R. \& Capecchi, M. R. Site-directed mutagenesis by gene targeting in mouse embryo-derived stem cells. Cell 6, 503-512 (1987).

4. Fauser, F. et al. In planta gene targeting. Proc. Natl. Acad. Sci. USA 109, 7535-7540 (2012).

5. Doudna, J. A. \& Charpentier, E. Genome editing. The new frontier of genome engineering with CRISPR-Cas9. Science 28, 1258096 (2014).

6. Cox, D. B., Platt, R. J. \& Zhang, F. Therapeutic genome editing: prospects and challenges. Nat. Med. 21, 121-131 (2015).

7. Weeks, D. P., Spalding, M. H. \& Yang, B. Use of designer nucleases for targeted gene and genome editing in plants. Plant Biotechnol. J. 14, 483-495 (2016).

8. Paszkowski, J., Baur, M., Bogucki, A. \& Potrykus, I. Gene targeting in plants. EMBO J. 7, 4021-4026 (1988).

9. Offringa, R. et al. Extrachromosomal homologous recombination and gene targeting in plant cells after Agrobacterium mediated transformation. EMBO J. 9, 3077-3084 (1990).

10. Terada, R., Urawa, H., Inagaki, Y., Tsugane, K. \& Iida, S. Efficient gene targeting by homologous recombination in rice. Nat. Biotechnol. 20, 1030-1034 (2002).

11. Shimatani, Z., Nishizawa-Yokoi, A., Endo, M., Toki, S. \& Terada, R. Positivenegative-selection-mediated gene targeting in rice. Front. Plant Sci. 5, 748 (2014).

12. Gallego, M. E., Sirand-Pugnet, P. \& White, C. I. Positive-negative selection and T-DNA stability in Arabidopsis transformation. Plant Mol. Biol. 39, 83-93 (1999).

13. Wang, H. X. et al. Positive-negative selection for homologous recombination in Arabidopsis. Gene 272, 249-255 (2001).

14. Morton, R. \& Hooykaas, P. J. J. Gene replacement. Mol. Breed. 1, 123-132 (1995).

15. Paquet, D. et al. Efficient introduction of specific homozygous and heterozygous mutations using CRISPR/Cas9. Nature 533, 125-129 (2016).

16. Choulika, A., Perrin, A., Dujon, B. \& Nicolas, J. F. Induction of homologous recombination in mammalian chromosomes by using the I-SceI system of Saccharomyces cerevisiae. Mol. Cell. Biol. 15, 1968-1973 (1995).

17. Miki, D. et al. Efficient generation of diRNAs requires components in the posttranscriptional gene silencing pathway. Sci. Rep. 7, 301 (2017).

18. Puchta, H., Dujon, B. \& Hohn, B. Two different but related mechanisms are used in plants for the repair of genomic double-strand breaks by homologous recombination. Proc. Natl. Acad. Sci. USA 93, 5055-5060 (1996).

19. Baltes, N. J., Gil-Humanes, J., Cermak, T., Atkins, P. A. \& Voytas, D. F. DNA replicons for plant genome engineering. Plant Cell 26, 151-163 (2014).

20. de Pater, S., Pinas, J. E., Hooykaas, P. J. \& van der Zaal, B. J. ZFN-mediated gene targeting of the Arabidopsis protoporphyrinogen oxidase gene through Agrobacterium-mediated floral dip transformation. Plant Biotechnol. J. 11, 510-515 (2013).

21. Qi, Y. et al. Increasing frequencies of site-specific mutagenesis and gene targeting in Arabidopsis by manipulating DNA repair pathways. Genome Res. 23, 547-554 (2013).

22. Schiml, S., Fauser, F. \& Puchta, H. The CRISPR/Cas system can be used as nuclease for in planta gene targeting and as paired nickases for directed mutagenesis in Arabidopsis resulting in heritable progeny. Plant J. 80, 1139-1150 (2014).

23. Weinthal, D. M., Taylor, R. A. \& Tzfira, T. Nonhomologous end joiningmediated gene replacement in plant cells. Plant Physiol. 162, 390-400 (2013).

24. Zhao, Y. et al. An alternative strategy for targeted gene replacement in plants using a dual-sgRNA/Cas9 design. Sci. Rep. 6, 23890 (2016). 
25. Cai, C. Q. et al. Targeted transgene integration in plant cells using designed zinc finger nucleases. Plant Mol. Biol. 69, 699-709 (2009).

26. Li, J. F. et al. Multiplex and homologous recombination-mediated genome editing in Arabidopsis and Nicotiana benthamiana using guide RNA and Cas9. Nat. Biotechnol. 31, 688-691 (2013).

27. Schaeffer, S. M. \& Nakata, P. A. The expanding footprint of CRISPR/Cas9 in the plant sciences. Plant Cell Rep. 35, 1451-1468 (2016).

28. Townsend, J. A. et al. High-frequency modification of plant genes using engineered zinc-finger nucleases. Nature 459, 442-445 (2009).

29. Wright, D. A. et al. High-frequency homologous recombination in plants mediated by zinc-finger nucleases. Plant J. 44, 693-705 (2005).

30. Zhang, Y. et al. Transcription activator-like effector nucleases enable efficient plant genome engineering. Plant Physiol. 161, 20-27 (2013).

31. Li, Z. et al. Cas9-guide RNA directed genome editing in soybean. Plant Physiol. 169, 960-970 (2015).

32. Čermák, T., Baltes, N. J., Čegan, R., Zhang, Y. \& Voytas, D. F. High-frequency, precise modification of the tomato genome. Genome Biol. 16, 232 (2015).

33. Filler Hayut, S., Melamed Bessudo, C. \& Levy, A. A. Targeted recombination between homologous chromosomes for precise breeding in tomato. Nat. Commun. 8, 15605 (2017)

34. Begemann, M. B. et al. Precise insertion and guided editing of higher plant genomes using Cpf1 CRISPR nucleases. Sci. Rep. 14, 11606 (2017).

35. Endo, M., Mikami, M. \& Toki, S. Biallelic gene targeting in rice. Plant Physiol. 170, 667-677 (2016).

36. $\mathrm{Li}$, J. et al. Gene replacements and insertions in rice by intron targeting using CRISPR-Cas9. Nat. Plants 12, 16139 (2016).

37. Li, T., Liu, B., Chen, C. Y. \& Yang, B. TALEN-mediated homologous recombination produces site-directed DNA base change and herbicideresistant rice. J. Genet. Genom. 43, 297-305 (2016).

38. Shan, Q. et al. Rapid and efficient gene modification in rice and Brachypodium using TALENs. Mol. Plant 6, 1365-1368 (2013).

39. Shan, Q., Wang, Y., Li, J. \& Gao, C. Genome editing in rice and wheat using the CRISPR/Cas system. Nat. Protoc. 9, 2395-2410 (2014).

40. Sun, Y. et al. Engineering herbicide-resistant rice plants through CRISPR/ Cas9-mediated homologous recombination of acetolactate synthase. Mol. Plant 9, 628-631 (2016).

41. Wang, M. et al. Gene targeting by homology-directed repair in rice using a geminivirus-based CRISPR/Cas9 system. Mol. Plant 10, 1007-1010 (2017).

42. Ainley, W. M. et al. Trait stacking via targeted genome editing. Plant Biotechnol. J. 11, 1126-1134 (2013).

43. Shi, J. et al. ARGOS8 variants generated by CRISPR-Cas9 improve maize grain yield under field drought stress conditions. Plant Biotechnol. J. 15, 207-216 (2017).

44. Shukla, V. K. et al. Precise genome modification in the crop species Zea mays using zinc-finger nucleases. Nature 459, 437-441 (2009).

45. Svitashev, S., Schwartz, C., Lenderts, B., Young, J. K. \& Mark Cigan, A. Genome editing in maize directed by CRISPR-Cas9 ribonucleoprotein complexes. Nat. Commun. 7, 13274 (2016).

46. Svitashev, S. et al. Targeted mutagenesis, precise gene editing, and site-specific gene insertion in maize using Cas9 and guide RNA. Plant Physiol. 169, 931-945 (2015).

47. Gil-Humanes, J. et al. High-efficiency gene targeting in hexaploid wheat using DNA replicons and CRISPR/Cas9. Plant J. 89, 1251-1262 (2017).

48. Wang, Y. et al. Simultaneous editing of three homoeoalleles in hexaploid bread wheat confers heritable resistance to powdery mildew. Nat. Biotechnol. 32, 947-951 (2014)

49. Butler, N. M., Baltes, N. J., Voytas, D. F. \& Douches, D. S. Geminivirusmediated genome editing in potato (Solanum tuberosum L.) using sequencespecific nucleases. Front. Plant Sci. 7, 1045 (2016).

50. Budhagatapalli, N., Rutten, T., Gurushidze, M., Kumlehn, J. \& Hensel, G. Targeted modification of gene function exploiting homology-directed repair of TALEN-mediated double-strand breaks in barley. G3 (Bethesda) 5, 1857-1863 (2015).

51. Sauer, N. J. et al. Oligonucleotide-mediated genome editing provides precision and function to engineered nucleases and antibiotics in plants. Plant Physiol. 170, 1917-1928 (2016).

52. D'Halluin, K. et al. Targeted molecular trait stacking in cotton through targeted double-strand break induction. Plant Biotechnol. J. 11, 933-941 (2013).

53. Mao, Y. et al. Development of germ-line-specific CRISPR-Cas9 systems to improve the production of heritable gene modifications in Arabidopsis. Plant Biotechnol. J. 14, 519-532 (2016).
54. Wang, Z. P. et al. Egg cell-specific promoter-controlled CRISPR/Cas9 efficiently generates homozygous mutants for multiple target genes in Arabidopsis in a single generation. Genome Biol. 16, 144 (2015).

55. Yan, L. et al. High-efficiency genome editing in Arabidopsis using YAO promoter-driven CRISPR/Cas9 system. Mol. Plant 8, 1820-1823 (2015).

56. Steffen, J. G. et al. Identification of genes expressed in Arabidopsis female gametophyte. Plant J. 51, 281-292 (2007).

57. Stevens, R. et al. A CDC homolog in Arabidopsis is essential for meiosis, as shown by RNA interference-induced gene silening. Plant Cell 16, 99-113 (2004).

58. Qian, W. et al. A histone acetyltransferase regulates active DNA demethylation in Arabidopsis. Science 336, 1445-1448 (2012).

59. Choi, Y. et al. DEMETER, a DNA glycosylase domain protein, is required for endosperm gene imprinting and seed viability in Arabidopsis. Cell 110, 33-42 (2002).

60. Duan, C. G. et al. MET18 connects the cytosolic iron-sulfur cluster assembly pathway to active DNA demethylation in Arabidopsis. PLoS Genet 11, e1005559 (2015).

61. Mok, Y. G. et al. Domain structure of the DEMETER 5-methylcytosine DNA glycosylase. Proc. Natl. Acad. Sci. USA 107, 19225-19230 (2010).

62. Lieberman-Lazarovich, M., Melamed-Bessudo, C., de Pater, S. \& Levy, A. A. Epigenetic alterations at genomic loci modified by gene targeting in Arabidopsis thaliana. PLoS ONE 8, e85383 (2013).

63. Bechtold, N. et al. The endosperm and the embryo of Arabidopsis thaliana are independently transfromed though infiltration by Agrobacterium tumefaciens. Transgenic Res. 12, 509-517 (2003).

\section{Acknowledgements}

This work was supported by the Chinese Academy of Sciences to J.-K.Z., and by Grant for Basic Science Research Projects from The Sumitomo Foundation to D.M. We would like to thank Life Science Editors for editorial assistance, Ms. Wencan Zhang for assistance, the Plant Cell Biology Core Facility at the Shanghai Center for Plant Stress Biology for assistance with confocal microscopy.

\section{Author contributions}

D.M., W.X.Z., and J.-K.Z. designed the research; W.X.Z. and D.M. performed the experiments with assistance from W.J.Z., and Z.F.; D.M. and J.-K.Z. supervised the project; D.M., W.X.Z. and J.-K.Z. wrote the paper.

\section{Additional information}

Supplementary Information accompanies this paper at https://doi.org/10.1038/s41467018-04416-0.

Competing interests: The authors declare no competing interests.

Reprints and permission information is available online at http://npg.nature.com/ reprintsandpermissions/

Publisher's note: Springer Nature remains neutral with regard to jurisdictional claims in published maps and institutional affiliations.

Open Access This article is licensed under a Creative Commons Attribution 4.0 International License, which permits use, sharing, adaptation, distribution and reproduction in any medium or format, as long as you give appropriate credit to the original author(s) and the source, provide a link to the Creative Commons license, and indicate if changes were made. The images or other third party material in this article are included in the article's Creative Commons license, unless indicated otherwise in a credit line to the material. If material is not included in the article's Creative Commons license and your intended use is not permitted by statutory regulation or exceeds the permitted use, you will need to obtain permission directly from the copyright holder. To view a copy of this license, visit http://creativecommons.org/ licenses/by/4.0/

(C) The Author(s) 2018 